\title{
Enhancement of Effective Electro-optic Coefficient in Domain Engineered UV-written Waveguides in $\mathrm{LiNbO}_{3}$
}

\author{
C. Y. J. Ying, ${ }^{1, *}$ G. Zisis, ${ }^{1}$ A. R. Naylor,${ }^{1}$ P. Ganguly, ${ }^{2}$ C. L. Sones, ${ }^{1}$ \\ E. Soergel, ${ }^{3}$ R. W. Eason ${ }^{1}$ and S. Mailis ${ }^{1}$ \\ ${ }^{1}$ Optoelectronics Research Centre, University of Southampton, Highfield, Southampton, SO17 1BJ, UK \\ ${ }^{2}$ Advanced Technology Development Centre, Indian Institute of Technology, Kharagpur 721302, India \\ ${ }^{3}$ Institute of Physics, University of Bonn, Wegelerstr. 8, 53115 Bonn, Germany \\ *yoy@orc.soton.ac.uk
}

\begin{abstract}
UV laser-induced poling-inhibition produces inverted domains in $\mathrm{LiNbO}_{3}$ which overlap significantly with waveguide modes. We have observed a $26 \%$ enhancement of the effective electro-optic coefficient in such domain-engineered waveguides.

OCIS codes: (130.3730) Lithium niobate; (130.0130) Integrated optics; (230.0230) Optical devices
\end{abstract}

\section{Introduction}

Lithium niobate crystal (LN) is a very important technological material which is widely used by the photonics industry today due to its significant optical nonlinearity and wide transparent window [1]. It also shows a significant electro-optic (EO) response which enables the fabrication of low-voltage operation, high speed integrated optical modulators that are routinely used in optical telecommunication and integrated optics [1]. For the fabrication of integrated optical circuits in this material it is necessary to produce channel waveguides which are commonly fabricated by Ti-indiffusion [1] or proton exchange [1]. However, a UV laser direct writing method for the fabrication of optical channel waveguides has been proposed recently [2]. The fabrication details and the electrooptic behavior of these waveguides have been reported in $[3,4]$.

Here we report on the enhancement of the electro-optic response of UV laser-written LN waveguides as a result of a post-poling process. More specifically we have observed a $26 \%$ increase of the $r_{33}$ coefficient compared to the bulk in UV-written LN waveguides that has been subjected to poling inhibition [5]. Poling inhibition produces inverted ferroelectric domains which are only a few microns deep. These domains are formed exactly in the same place as the UV written tracks which are responsible for the waveguide formation, and they overlap significantly with the propagating waveguide mode as is illustrated schematically in Fig. 1. Due to the polarization-selective transmission in the UV-written waveguides only the $r_{33}$ coefficient could be investigated.
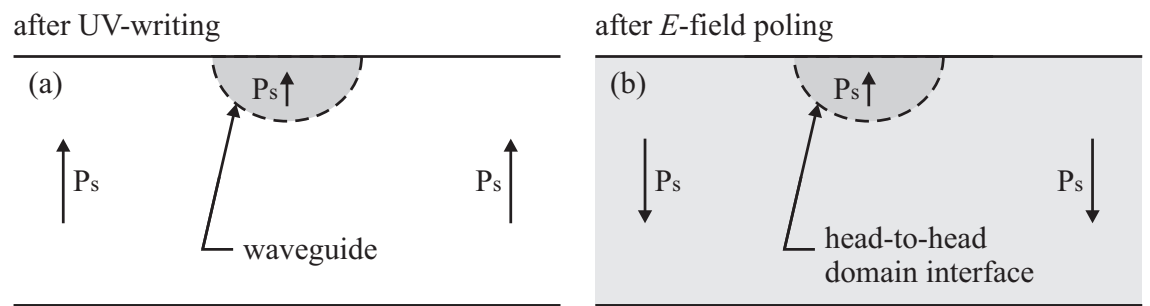

Fig. 1. Schematic of the cross section of a) a UV-written waveguide on a single domain substrate, and b) the head-to-head domain arrangement overlapping with the waveguide after poling-inhibition.

\section{Experiment}

Optical channel waveguides were fabricated by direct UV laser writing on the $+z$ face of a $0.5 \mathrm{~mm}$-thick, $z$-cut undoped congruent lithium niobate substrate by scanning a focused c.w. frequency-doubled argon ion laser (244nm) across the surface of the crystal as described in [4]. The focused beam diameter was $\sim 7-8 \mu \mathrm{m}$, the writing speed was $0.1 \mathrm{~mm} / \mathrm{s}$, and the writing power was $45 \mathrm{~mW}$. The sample was subsequently subjected to electric field poling (EFP) using an externally applied electric field $(\sim 19.5 \mathrm{kV} / \mathrm{mm})$ which resulted in local poling-inhibited domains of limited depth that overlap with the waveguides as shown in Fig. 1b [5,6].

The electro-optic response was evaluated interferometrically by placing the waveguides in one branch of a Mach-Zehnder interferometer as described in [3]. Both $z$-faces of the lithium niobate waveguide substrate were 
covered by a thin (20nm) gold film in order to apply an electric field along the $z$-axis for the evaluation of the $r_{33}$ coefficient. The experimental setup which was used for the measurements is schematically illustrated in Fig. 2.

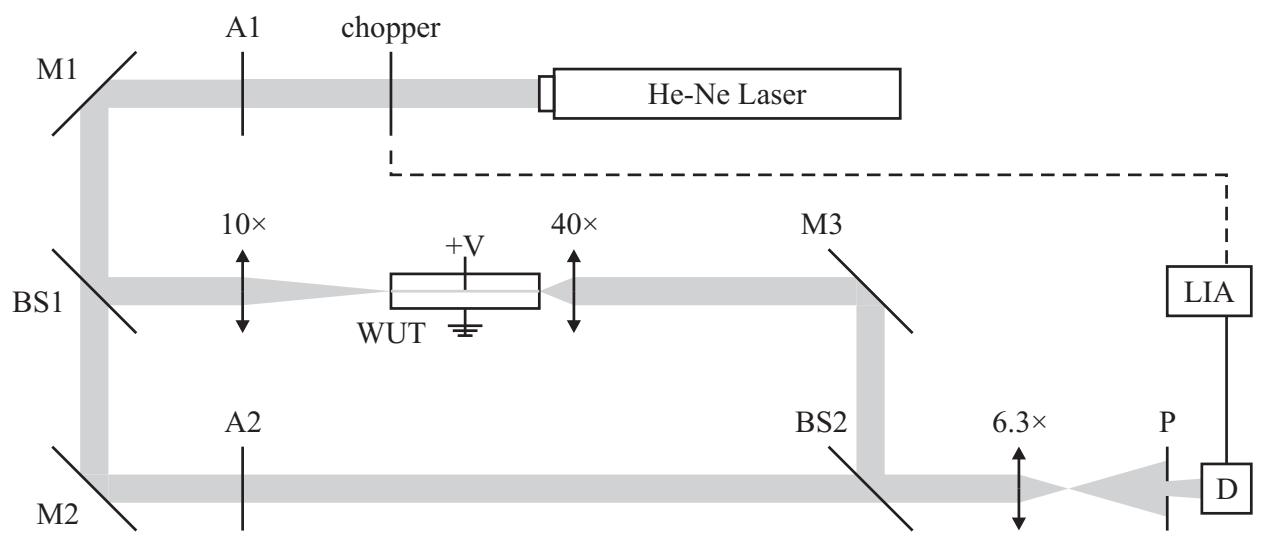

Fig. 2. Experimental setup for the EO coefficient measurement of the waveguide. $(\mathrm{A} 1, \mathrm{~A} 2=$ optical attenuator; $\mathrm{M} 1, \mathrm{M} 2, \mathrm{M} 3=\mathrm{mirrors}$; BS1, BS2 = beam splitter; WUT = waveguide under test; $\mathrm{V}=$ applied voltage; $\mathrm{P} 1, \mathrm{P} 2=$ pinhole; $\mathrm{D}=$ detector; LIA = lock-in amplifier.)

The EO phase shift in the waveguide sample was measured by monitoring the movement of the interference fringes in the output of the interferometer as a function of the voltage applied to the sample. The half-wave voltage $V_{\pi}$ was measured in this way and the $r_{33}$ coefficient was derived using the expression $r_{33}=\lambda d n_{e f f}^{-3} L^{-1} V_{\pi}^{-1}$ [3], where $\lambda$ is the operating wavelength, in our case $632.8 \mathrm{~nm} ; d$ is the substrate thickness $(0.5 \mathrm{~mm})$; $n_{\text {eff }}$ is the effective refractive index of the waveguide; and $L$ is the length of the electrode-covered waveguide section. A set of titanium in-diffused waveguides was used as a control sample to provide the background measurement of the bulk for the $r_{33}$ coefficient.

\section{Results and discussion}

The measured values of the electro-optic coefficient $\left(r_{33}\right)$ in the poling-inhibited samples proved to be systematically higher than the value obtained with the control sample of unpoled titanium in-diffused waveguides which was $35 \mathrm{pm} / \mathrm{V}$. The highest value of the $r_{33}$ that was measured in the poling-inhibited waveguides was $44.2 \mathrm{pm} / \mathrm{V}$ which corresponds to an enhancement of $26 \%$ compared to reference sample, therefore the bulk. The observed enhancement in the value of the EO coefficient is attributed to the strain which is associated with the presence of a head-to-head domain boundary that surrounds the optical waveguide channel as illustrated in Fig. 1b. The enhancement of the EO coefficient varied for waveguides which were fabricated under different UV irradiation conditions. The irradiation conditions affect both the waveguide mode confinement and the depth of the polinginhibited domains. This suggests that the enhancement can be further optimized and even applied to other waveguide systems such as titanium in-diffused and proton exchanged channel guides.

\section{Conclusion}

In conclusion, a $26 \%$ enhancement of the $r_{33}$ coefficient in UV-written waveguides has been observed in UV laser written lithium niobate waveguides which have been domain engineered by inhibition of poling. The poling inhibition process produces an inverted domain that overlaps with the waveguide mode and an associated domain wall that surrounds the optical waveguide. The strain across the head-to-head domain wall is suggested here to be responsible for the observed enhancement of the EO coefficient.

[1] K. K. Wong, Properties of Lithium Niobate (The Institution of Engineering and Technology, 2002).

[2] S. Mailis, C. Riziotis, I. T. Wellington, P. G. R. Smith, C. B. E. Gawith and R. W. Eason, "Direct UV writing of channel waveguides in congruent lithium niobate single crystals," Opt. Lett. 28, 1433-1435 (2003).

[3] C. L. Sones, P. Ganguly, Y. J. Ying, F. Johann, E. Soergel, R. W. Eason and S. Mailis, "Spectral and electro-optic response of UV-written waveguides in $\mathrm{LiNbO}_{3}$ single crystals," Opt. Express 17, 23755-23764 (2009).

[4] P. Ganguly, C. L. Sones, Y. J. Ying, H. Steigerwald, K. Buse, E. Soergel, R. W. Eason, S. Mailis, "Determination of refractive indices from the mode profiles of UV-written channel waveguides in $\mathrm{LiNbO}_{3}$-crystals for optimization of writing conditions," IEEE Journal of Lightwave Technology 27, 3490-3497 2009

[5] C. L. Sones, A. C. Muir, Y. J. Ying, S. Mailis, R. W. Eason, T. Jungk, A. Hoffmann and E. Soergel, "Precision nanoscale domain engineering of lithium niobate via UV laser induced inhibition of poling," Appl. Phys. Lett. 92, 072905 (2008).

[6] C. L. Sones, P. Ganguly, Y. J. Ying, E. Soergel, R. W. Eason and S. Mailis, "Poling-inhibited ridge waveguides in lithium niobate crystals," Appl. Phys. Lett. 97, 151112 (2010). 